\title{
Surface Bloch waves in metamaterial and metal-dielectric superlattices
}

\author{
Slobodan M. Vukovic, ${ }^{1,2}$ Ilya V. Shadrivov, ${ }^{1, a)}$ and Yuri S. Kivshar ${ }^{1}$ \\ ${ }^{1}$ Nonlinear Physics Center, Research School of Physics and Engineering, Australian National University, \\ Canberra, Australian Capital Territory 0200, Australia \\ ${ }^{2}$ Institute of Physics, University of Belgrade, Zemun 11080, Serbia
}

(Received 10 March 2009; accepted 26 June 2009; published online 28 July 2009)

\begin{abstract}
We study the properties of electromagnetic Bloch waves in semi-infinite periodic structures created by alternating metamaterial and dielectric layers. We derive and analyze the dispersion relations in the long-wavelength limit for both TE- and TM-polarized surface Bloch modes for magnetic metamaterials with negative refraction and metal-dielectric plasmonic superlattices. We reveal that in the subwavelength regime, the bulk modes are characterized by three different refractive indices ("trirefringence"), while the surface modes can propagate parallel to the Bloch wavevector and along the interface between superlattice and semi-infinite dielectric. () 2009 American Institute of Physics. [DOI: 10.1063/1.3186041]
\end{abstract}

Subwavelength confinement and manipulation of light in nanoscale optical structures are of a great importance for applications in imaging, sensing, high-resolution lithography, all-optical signal processing, photonic funnels, and superlenses. ${ }^{1-6}$ However, the diffraction limit prevents the light confinement on the scales smaller than a half of the wavelength. Conventional techniques for the resolution improvement are based on the use of high-index materials, ${ }^{7}$ but the further progress in this field requires a design of artificial materials, often termed as metamaterials, with higher refractive indices. ${ }^{8,9}$ Plasmonic macrostructure composites that operate in the regime of coupled waveguide modes have been recently proposed for a variety of future imaging systems and beam steering. ${ }^{10-13}$ At the same time, the absence of natural magnetism at optical and infrared frequencies requires again the fabrication of nanostructured metamaterials in order to achieve required dispersion properties for negative refraction. ${ }^{14-17}$ A typical width of a single layer in such metamaterials is of the order of $10 \mathrm{~nm}$ that is much smaller than the free-space wavelength of optical or infrared radiation. Thus, it is usually assumed that optical properties of those multilayered composites can be described by an effective-medium approximation, ${ }^{18}$ although the typical field variation length may be less than the free-space wavelength. ${ }^{19}$ On the other hand, many studies of negativeindex materials that use the original design based on simultaneously negative dielectric permittivity and magnetic permeability assume that the homogenization limit for metamaterials is fulfilled either for isotropic ${ }^{20-22}$ or "indefinite" 23 models. In particular, the effective-medium approximation has been used to study surface waves at the interfaces between metamaterial and surrounding media that may lead to the subdiffraction imaging.

In this letter, we study the electromagnetic waves propagating in two-dimensional semi-infinite periodic structure created by alternating layers of metamaterial and conventional dielectric and semi-infinite homogeneous dielectric placed normally to the interfaces within the multilayered media [see the geometry in Fig. 1(a)]. A unit cell of this layered structure is formed by two layers: one with the thickness $a_{1}$,

${ }^{a)}$ Electronic mail: ivs124@rsphysse.anu.edu.au. dielectric permittivity $\varepsilon_{1}$, and magnetic permeability $\mu_{1}$, and the other one with parameters $a_{2}, \varepsilon_{2}$, and $\mu_{2}$, respectively. The layered media have the period $d=a_{1}+a_{2}$. Such a structure may support the propagation of surface waves in the direction parallel to the Bloch wavevector $k_{B} \| z$ in the layered medium. Surface waves that propagate along the interface between the layered dielectric media and a semi-infinite dielectric cladding placed parallel to the interfaces within the multilayered medium (i.e., with the wavevector $k_{\perp} \| x$ perpendicular to the Bloch wavevector) were studied more than 30 years ago in Refs. 24 and 25. Although many authors have investigated subsequently the same geometry, the surface waves that propagate parallel to the Bloch wavevector have not been analyzed yet to the best of our knowledge. We term those waves as surface Bloch waves.

There exist surface modes supported by each individual interface within the layered media, but the coupling (weak or strong) of these modes gives birth to new collective modes that propagate in the same direction, i.e., perpendicular to the Bloch wavevector. In the regime of strong coupling, when the unit cell thickness $d$ is much smaller than the free-space wavelength $\lambda$, the optical properties of a multilayered structure can be described by effective permittivity ${ }^{18} \hat{\varepsilon}$ and effective permeability $\hat{\mu}$. Generally speaking, these quantities are diagonal tensors with two equal components in the directions perpendicular to the Bloch wavevector: $\hat{\varepsilon}=\operatorname{diag}\left(\varepsilon_{\perp}, \varepsilon_{\perp}, \varepsilon_{\|}\right)$ and $\hat{\mu}=\operatorname{diag}\left(\mu_{\perp}, \mu_{\perp}, \mu_{\|}\right)$.

Assuming that the electric field of the $s$-polarized (TE) mode and the magnetic field of the $p$-polarized (TM) mode are in the form $\sim A_{k_{B}}(z) \exp \left(i k_{B} z+i k_{\perp} x-i \omega t\right) \mathbf{y}$, the dispersion (a)

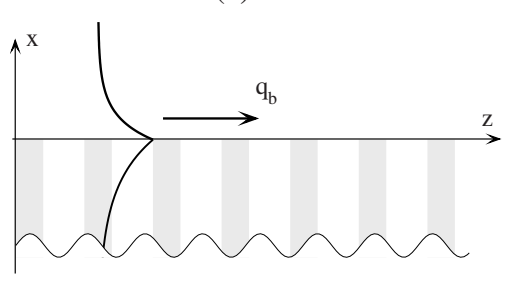

(b)

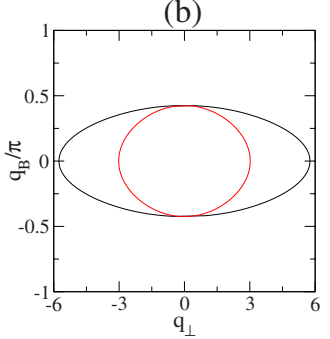

FIG. 1. (Color online) (a) Geometry of the problem. (b) Equifrequency contours for $\omega / \omega_{p}=0.3$ obtained from the dispersion (2). 
of the Bloch modes in the infinite layered media is described by the well-known equation for bulk Bloch waves that comes from the eigenvalue problem for two-layer transfer matrix, ${ }^{24,25}$

$$
\begin{aligned}
\cos \left(q_{B} d\right)= & \cos \left(k_{1} a_{1}\right) \cos \left(k_{2} a_{2}\right) \\
& -\frac{1+\alpha_{s, p}^{2}}{2 \alpha_{s, p}} \sin \left(k_{1} a_{1}\right) \sin \left(k_{2} a_{2}\right),
\end{aligned}
$$

where $k_{1,2}=\left(\varepsilon_{1,2} \mu_{1,2}-q_{\perp}^{2}\right)^{1 / 2}$. We note that all spatial dimensions are normalized to $c / \omega$ and wavenumbers to $\omega / c$. The polarization dependent coefficients $\alpha_{s, p}$ are defined as $\alpha_{s}$ $=k_{2} \mu_{1} / k_{1} \mu_{2}$ and $\alpha_{p}=k_{2} \varepsilon_{1} / k_{1} \varepsilon_{2}$. In the subwavelength regime, when $\left|k_{1} a_{1}\right| \ll 1$ and $\left|k_{2} a_{2}\right| \ll 1$, after the Taylor expansion in Eq. (1), we obtain the dispersion relations similar to those for the light propagation in uniaxial crystals, for the $s$ - and $p$-polarized waves,

$$
\frac{\sin ^{2} q_{B} d / 2}{n_{o}^{2}}+\frac{q_{\perp}^{2}(d / 2)^{2}}{\left(n_{e}^{s, p}\right)^{2}}=\frac{d^{2}}{4},
$$

where $n_{o}=\sqrt{\varepsilon_{\perp} \mu_{\perp}}$ is the refractive index of the ordinary wave and $n_{e}^{s}=\sqrt{\varepsilon_{\perp} \mu_{\|}}$and $n_{e}^{p}=\sqrt{\varepsilon_{\|} \mu_{\perp}}$ are the refractive indices of $s$ - and $p$-polarized extraordinary waves, respectively. The effective material parameters appear as a result of the Taylor expansion rather than an averaging procedure,

$$
\begin{aligned}
& \varepsilon_{\perp}=(1-\delta) \varepsilon_{1}+\delta \varepsilon_{2} ; \quad \varepsilon_{\|}=\frac{\varepsilon_{1} \varepsilon_{2}}{(1-\delta) \varepsilon_{2}+\delta \varepsilon_{1}}, \\
& \mu_{\perp}=(1-\delta) \mu_{1}+\delta \mu_{2} ; \quad \mu_{\|}=\frac{\mu_{1} \mu_{2}}{(1-\delta) \mu_{2}+\delta \mu_{1}},
\end{aligned}
$$

where $\delta=a_{2} / d, 0<\delta<1$, describes the degree of anisotropy. The structure becomes isotropic at $\delta=0$ and $\delta=1$ and anisotropic between these values.

Equation (2) describes the dispersion relations of bulk waves for infinite layered media in the long-wavelength approximation. For nonmagnetic media, and under the additional approximation $\left|q_{B} d\right| \ll 1$, these equations reduce to the equations obtained in Refs. 24 and 25, where it was shown that a periodic stratified medium is formally analogous to an uniaxial crystal, yielding for $s$ - and $p$-polarizations, respectively: $q_{B}^{2}+q_{\perp}^{2}=\varepsilon_{\perp}$ and $q_{B}^{2} / \varepsilon_{\perp}+q_{\perp}^{2} / \varepsilon_{\|}=1$. Although these equations look very attractive (because they are formally analogous to the well-known Fresnel equations), it is worth noting that imposing $\left|q_{B} d\right| \ll 1$ leads to loosing zonal structure and band gaps typical for Bloch waves. Under the same approximation, the inclusion of higher order terms in the Taylor expansion revealed nonlocal effects (spatial dispersion) in nanolayered metamaterials. ${ }^{19}$ On the other hand, the inclusion of magnetic metamaterials gives rise to the appearance of the extraordinary wave even in the case of $s$-polarization if the formal analogy with uniaxial crystals has been adopted. There are two ordinary $s$ - and $p$-polarized modes that obey the same dispersion law, $\sin ^{2} q_{B} d / 2$ $=\varepsilon_{\perp} \mu_{\perp}(d / 2)^{2}$, and two extraordinary $s$ - and $p$-polarized modes with different dispersions, $q_{\perp}^{2}=\varepsilon_{\perp} \mu_{\|}$and $q_{\perp}^{2}=\varepsilon_{\|} \mu_{\perp}$, respectively. Thus, for the same frequency, we now have three different refractive indices, and when $\left|q_{B} d\right| \ll 1$, Eq. (2) represents two ellipses in $\left(q_{B}, q_{\perp}\right)$ plane with one common axis [see Fig. 1(b)] instead of a circle and an ellipse, which we have for uniaxial crystals. So, magnetic metamaterials

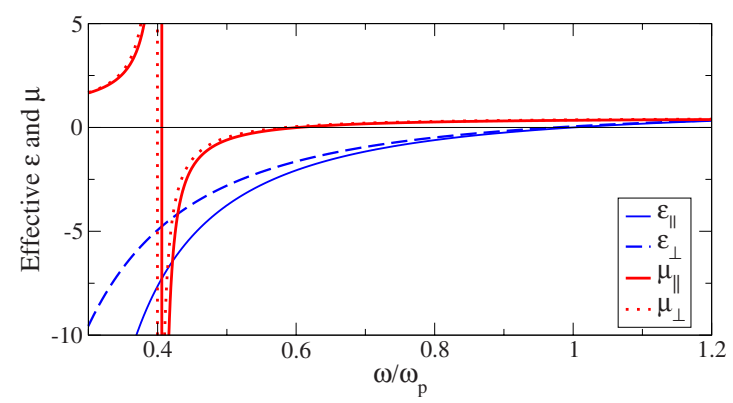

FIG. 2. (Color online) Effective dielectric and magnetic parameters of the metamaterial superlattices $(\delta=0.95)$.

can produce either negative or normal (positive) refraction in all directions, as well as negative refraction in one direction and normal in the other one. We emphasize that for both $s$ and $p$-polarizations, the desired characteristics of the periodic stratified metamaterials can be engineered by a variation in the thicknesses of the constitutive layers.

As an example, in Fig. 2 we plot the effective parameters for the case of a periodic structure with metamaterial layers in vacuum $\left(\varepsilon_{1}=\mu_{1}=1\right)$. We assume that the metamaterial layers have the frequency-dependent dielectric permittivity and magnetic permeability of the forms $\varepsilon_{2}=1-\omega_{p}^{2} / \omega^{2}$ and $\mu_{2}=1-F \omega^{2} /\left(\omega^{2}-\omega_{g}^{2}\right)$, where we take $F=0.56$ and $\omega_{g}^{2} / \omega_{p}^{2}$ $=0.16$.

To study surface Bloch waves in long-wavelength limit, we assume that multilayered media are truncated, and it occupies a half-space $x<0$, while the other half-space $(x>0)$ is occupied by homogeneous dielectric with dielectric constant $\varepsilon_{c}$. To obtain the solutions for surface waves, we consider in Eq. (2) purely imaginary values of $q_{\perp}$. Using the standard continuity conditions of the tangential field components, we then obtain the dispersion relation for the $s$-polarized surface waves,

$$
\sqrt{\frac{\mu_{\|}}{\mu_{\perp}}\left[\sin ^{2}\left(q_{B} d / 2\right)-\varepsilon_{\perp} \mu_{\perp}(d / 2)^{2}\right]}=-\mu_{\|} \frac{d}{2} \sqrt{q_{B}^{2}-\varepsilon_{c}},
$$

whereas for the $p$-polarized surface waves, we have

$$
\varepsilon_{c} \sqrt{\frac{\varepsilon_{\|}}{\varepsilon_{\perp}}\left[\sin ^{2}\left(q_{B} d / 2\right)-\varepsilon_{\perp} \mu_{\perp}(d / 2)^{2}\right]}=-\varepsilon_{\|} \frac{d}{2} \sqrt{q_{B}^{2}-\varepsilon_{c}} .
$$

Equations (4) and (5) are obtained in the approximation that the thicknesses of each layer is small; however we did not make any assumptions about the values for the Bloch wavenumber. However, since we used continuous medium approximation for matching boundary conditions on the interface, it is expected that the results are valid when the Bloch wavelength is larger than the period of the unit cell $\left(q_{B} d\right.$ $<1)$. A more general case of arbitrary wavenumber will require numerical simulation in order to obtain dispersion properties of surface Bloch waves.

Now we calculate the dispersion of the surface Bloch modes with the effective parameters shown in Fig. 2 for the waves propagating along the interface between the structured medium and vacuum $\left(\varepsilon_{c}=1\right)$. Dispersion of surface Bloch modes for metamaterial superlattices created by layers of metamaterial and conventional dielectric is calculated using Eqs. (4) and (5), and it is shown in Fig. 3. From the dispersion equations we see that the $p$-polarized waves can exist in 


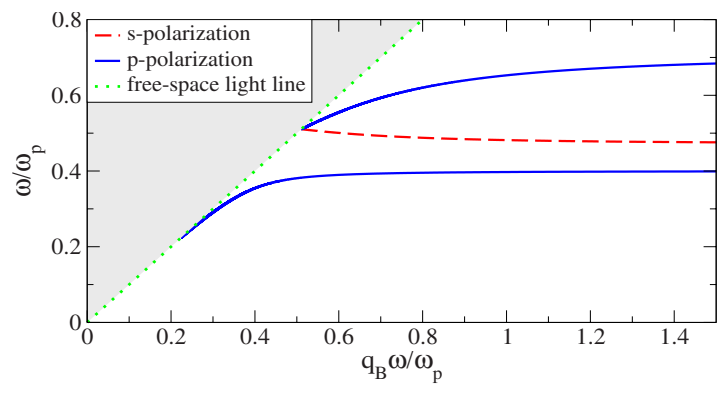

FIG. 3. (Color online) Dispersion of $s$ - and $p$-polarized Bloch surface waves in metamaterial superlattices for $\delta=0.95$ and $d=0.1 c / \omega_{p}$.

the frequency ranges where $\varepsilon_{\|}<0$, while $s$-polarized waves exist for $\mu_{\|}<0$. We found that for the values of the parameter $\delta$ close to one, there are in general two branches of $p$-polarized waves and one branch of $s$-polarized waves. Both upper and lower branches of the $p$-polarized waves are forward, while the $s$-polarized surface Bloch wave is backward (negative group velocity), and it exists in the range of frequencies of $0.4<\omega / \omega_{p}<0.603$. When we decrease the parameter $\delta$ toward the value of $1 / 2$, the frequency range for the $s$-polarized wave existence becomes narrower, and it becomes forward (not shown), and then it disappears. $P$-polarized waves also disappear for values of $\delta<1 / 2$, and there are no surface waves for such values of $\delta$ within the limits of our approximation.

For nonmagnetic metamaterials or metals in the layered structure, we use the Drude-type formula for $\varepsilon_{2}(\omega)$ but consider $\mu_{2}=1$. We find no $s$-polarized surface waves, but for the $p$-polarized surface Bloch modes we obtain the dispersion shown in Fig. 4. Surface Bloch modes exist only when the metal occupies more than half of the structure, i.e., $1 / 2$ $<\delta<1$. As we change the metal filling fraction from $\delta=1$, when the frequency range of the mode existence is 0 $<\omega / \omega_{p}<1 / \sqrt{2}$, to $\delta=1 / 2$, the frequency range of mode existence vanishes.

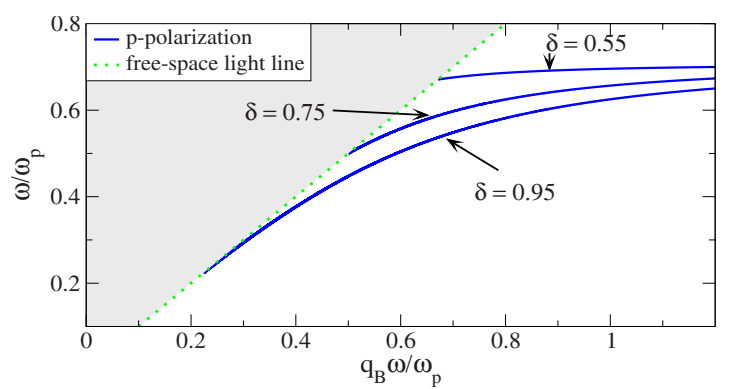

FIG. 4. (Color online) Dispersion of $p$-polarized Bloch surface waves in metal-dielectric structures for three different values of $\delta$ and $d=0.1 c / \omega_{p}$.
In conclusion, we have derived and analyzed the dispersion characteristics of the electromagnetic surface Bloch waves in metamaterial and metal-dielectric structures in the long-wavelength limit. We have revealed that in the subwavelength regime, the bulk modes are characterized by three different refractive indices, while the surface modes can propagate parallel to the Bloch wavevector along an interface between two superlattices. As a special case, we have studied the propagation along an interface between the superlattice and a semi-infinite dielectric in truncated layered structures. We note that the short-wavelength limit requires intensive numerical simulations, and it will be studied elsewhere.

The work was supported by the Australian Research Council. S.V. thanks Nonlinear Physics Center for hospitality and support during his stay in Canberra, and he acknowledges the support of the Serbian Ministry of Science (Grant No. OI 141031).

${ }^{1}$ J. B. Pendry, Phys. Rev. Lett. 85, 3966 (2000).

${ }^{2}$ N. Fang, H. Lee, C. Sun, and X. Zhang, Science 308, 534 (2005).

${ }^{3}$ H. Shin and S. Fan, Phys. Rev. Lett. 96, 073907 (2006).

${ }^{4}$ Z. Jacob, L. V. Alekseyev, and E. Narimanov, Opt. Express 14, 8247 (2006).

${ }^{5}$ D. R. Smith and J. B. Pendry, J. Opt. Soc. Am. B 23, 391 (2006).

${ }^{6} \mathrm{E}$. Ozbay, Science 311, 189 (2006).

${ }^{7}$ A. W. Fang, H. Park, O. Cohen, R. Jones, M. J. Paniccia, and J. E. Bowers, Opt. Express 14, 9203 (2006).

${ }^{8}$ A. Karalis, E. Lidorikis, M. Ibanescu, J. D. Joannopoulos, and M. Soljacic, Phys. Rev. Lett. 95, 063901 (2005).

${ }^{9}$ A. A. Govyadinov and V. A. Podolskiy, Phys. Rev. B 73, 155108 (2006).

${ }^{10}$ A. Alu and N. Engheta, IEEE Trans. Microwave Theory Tech. 52, 199 (2004).

${ }^{11}$ V. A. Podolskiy and E. E. Narimanov, Phys. Rev. B 71, 201101 (2005).

${ }^{12}$ R. Wangberg, J. Elser, E. E. Narimanov, and V. A. Podolskiy, J. Opt. Soc. Am. B 23, 498 (2006).

${ }^{13}$ T. J. Antosiewicz, W. M. Saj, J. Pniewski, and T. Szoplik, Opt. Express 14, 3389 (2006).

${ }^{14}$ I. V. Shadrivov, A. A. Sukhorukov, and Yu. S. Kivshar, Appl. Phys. Lett. 82, 3820 (2003).

${ }^{15}$ S. Zhang, W. Fan, N. C. Panoiu, K. J. Malloy, R. M. Osgood, and S. R. J. Brueck, Phys. Rev. Lett. 95, 137404 (2005).

${ }^{16}$ S. Zhang, W. Fan, K. J. Malloy, S. R. J. Brueck, N. C. Panoiu, and R. M. Osgood, Opt. Express 13, 4922 (2005).

${ }^{17}$ G. Dolling, C. Enkrich, M. Wegener, C. M. Soukoulis, and S. Linden, Science 312, 892 (2006).

${ }^{18}$ A. Alu and N. Engheta, J. Opt. Soc. Am. B 23, 571 (2006).

${ }^{19}$ J. Elser, V. A. Podolskiy, I. Salakhutdinov, and I. Avrutsky, Appl. Phys. Lett. 90, 191109 (2007).

${ }^{20}$ V. G. Veselago, Usp. Fiz. Nauk 92, 517 (1967) [Sov. Phys. Usp. 10, 509 (1968)].

${ }^{21}$ R. A. Shelby, D. R. Smith, and S. Schultz, Science 292, 77 (2001).

${ }^{22}$ I. V. Lindell, S. A. Tretyakov, K. I. Nikoskinen, and S. Ilvonen, Microwave Opt. Technol. Lett. 31, 129 (2001).

${ }^{23}$ D. R. Smith and D. Schurig, Phys. Rev. Lett. 90, 077405 (2003).

${ }^{24}$ P. Yeh, A. Yariv, and C. S. Hong, J. Opt. Soc. Am. 67, 423 (1977).

${ }^{25}$ A. Yariv and P. Yeh, J. Opt. Soc. Am. 67, 438 (1977). 